\title{
CONTROL DE ACELERACIONES DE ENTREPISO PARA SISTEMAS ESTRUCTURALES CON AISLAMIENTO SÍSMICO DE BASE
}

\author{
Oscar Zúñiga Cuevas ${ }^{(1)}$ y Amador Terán Gilmore ${ }^{(1)}$
}

\begin{abstract}
RESUMEN
Se evalúa la respuesta sísmica de una serie de sistemas simples con aislamiento sísmico de base para entender el efecto que las propiedades estructurales de dichos sistemas y de su super-estructura tienen en las demandas de aceleración de entrepiso. Se supuso que los sistemas estructurales permanecen elásticos y que la disipación de energía en el sistema de aislamiento puede modelarse como amortiguamiento viscoso. La diferencia entre los niveles de amortiguamiento de la superestructura y el sistema de aislamiento fue tomada en cuenta a través de un enfoque de amortiguamiento no-clásico. Para la evaluación de la respuesta dinámica de los sistemas se utilizaron movimientos del terreno registrados en sitios de suelo firme ubicados en la costa del Pacífico Mexicano. Una vez identificadas las propiedades estructurales que permiten el control de las demandas de aceleración de entrepiso, se discute el uso de un sistema equivalente de un grado de libertad para el diseño de sistemas estructurales de no más de cuatro niveles con aislamiento sísmico de base.
\end{abstract}

Palabras clave: Aislamiento sísmico; control de aceleración; amortiguamiento no-clásico

\begin{abstract}
The dynamic response of a series of base-isolated structures subjected to ground motions that were recorded on firm soils located in the Mexican Pacific coast was studied to understand the influence of their structural properties on the floor acceleration demands. The structural systems were considered elastic and the dissipating energy capacity of the isolation system was represented through a viscous damping model. The damping difference between the super-structure and the seismic isolation system was considered through a non-classical damping approach. Upon identifying the effect of the structural properties that are able to control the floor acceleration demands, an equivalent single-degree-of-freedom system is discussed to conceive base-isolation systems for low-rise structural systems.
\end{abstract}

Key words: Isolation system; acceleration control; non-classical damping; contents

Artículo recibido el 18 de noviembre de 2011 y aprobado para su publicación el 1 de abril de 2012

(1) Departamento de Materiales, Universidad Autónoma Metropolitana, Av. San Pablo 180, Colonia Reynosa Tamaulipas, México 02200, D.F. ozunigac@gmail.com, tga@correo.azc.uam.mx 


\section{INTRODUCCIÓN}

El mal desempeño que han exhibido algunas edificaciones y sus contenidos durante sismos recientes (Northridge 1994, Kobe 1995, Taiwan 1999, Sichuan 2008, Chile 2010), y el nivel tan alto de pérdidas sociales y económicas que esto representa, ha creado desconcierto en la comunidad internacional de ingeniería estructural. Dentro de un contexto donde el desempeño de los nuevos edificios debe satisfacer las múltiples y complejas necesidades de las sociedades modernas, se han planteado nuevos procedimientos de diseño con la aspiración de dar lugar a sistemas estructurales que sean capaces de controlar adecuadamente su nivel de daño cuando se les sujeta a excitaciones sísmicas con diferente intensidad. Considerando que el costo de los contenidos de estructuras altamente especializadas, como las que alojan hospitales, museos, centros de operación y otras estructuras esenciales, pueden a llegar a ser muy altos, se requieren enfoques de diseño que explícitamente consideren el control de las demandas de aceleración en los diferentes entrepisos de un sistema estructural.

El enfoque de diseño por desempeño ha aportado un marco para el planteamiento de metodologías de diseño innovadoras. Dentro de este contexto, el parámetro más utilizado para plantear el control de daño por sismo ha sido la demanda máxima de desplazamiento lateral (Bertero y Bertero 1992, Moehle 1992, MontielOrtega y Terán-Gilmore 2011, Priestley et al., 2007). Sin embargo, el control del desplazamiento lateral solo puede aspirar a controlar el daño en los sistemas estructural y no estructural, y en algunos tipos de contenidos. Bajo ciertas circunstancias, la falta de control de otros parámetros de respuesta, tal como la velocidad o aceleración absoluta, puede resultar en daño severo en los contenidos y por tanto, en pérdidas considerables (Chaudhuri y Hutchinson 2004, Medina et al. 2006, Poland y Hom 1997, Taghavi y Miranda 2003, Takahashi y Shiohara 2004, Villaverde 1997). Por tanto, solo a partir del control de la aceleración y velocidad de entrepiso podrá promoverse un desempeño adecuado para muchas estructuras esenciales. En términos de definición, se entiende por contenidos a aquellos elementos que no forman parte del sistema estructural principal; pero que pueden estar sujetos a fuerzas sísmicas importantes y dependen de sus características físicas para resistirlas. Respecto a lo anterior, Villaverde (1997) clasifica los contenidos en: 1) Componentes arquitectónicos; 2) Equipo mecánico y eléctrico; y 3) Contenidos típicos de los edificios (mobiliario).

Varios estudios experimentales y analíticos orientados a entender la interacción dinámica que se da entre los contenidos y los sistemas estructurales que los alojan establecen que las demandas de velocidad y aceleración en los contenidos dependen de múltiples variables, de tal forma que la formulación de una metodología de diseño para los contenidos requiere de planteamientos analíticos muy complejos (Kaneko et al. 2008, Medina et al. 2009, Medina et al. 2006, Sankaranarayanan 2007, Villaverde 2006). En términos generales, las demandas de aceleración en los contenidos dependen de: A) El tipo de sistema estructural y la variación en altura de su masa y propiedades estructurales; B) La ubicación en altura de los contenidos; y C) La interacción que ocurre entre las propiedades mecánicas y dinámicas de los sistemas estructurales y los contenidos; y la interacción que tengan ambos con los contenidos de frecuencia y energía del movimiento del terreno.

A la fecha, y a pesar de los esfuerzos prácticos y académicos por no dejar un vacío en cuanto al control del daño en contenidos, lo cierto es que la sobre-simplificación implícita en las metodologías actuales de diseño, y el hecho de que este diseño no forma parte explícita del proceso del diseño sísmico, resulta en muchos casos en contenidos altamente vulnerables que son expuestos a altas demandas de aceleración. En instalaciones estratégicas, como hospitales, esta situación resulta en la pérdida de operación en momentos muy críticos para una población que ha sufrido el embate de un sismo intenso. Lo anterior queda plenamente ilustrado a través del desempeño de las instalaciones hospitalarias durante los sismos de Northridge 1994 y Maule 2010 (Todd et al. 1994, Rojas et al. 2011, Elnashai et al.). Debido a esto, es urgente desarrollar 
metodologías de diseño sísmico que dentro de un marco de diseño por desempeño den lugar a sistemas estructurales que sean capaces de controlar el daño en sus contenidos a través del control explícito de la aceleración de entrepiso. Dentro de un enfoque de diseño basado en el balance demanda-capacidad, se han llevado a cabo recientemente múltiples estudios experimentales y analíticos encaminados a establecer umbrales de aceleración asociados a diferentes niveles de desempeño para diferentes tipos de contenidos (Comerio y Holmes 2004, Kaneko et al. 2008, Konstantinidis y Markis 2006, Marsantyo et al. 2000, Retamales et al. 2006). Lo anterior representa un intento por establecer las capacidades en términos de aceleración de los contenidos. Por el otro lado y desde el punto de vista de las demandas, urge desarrollar metodologías de diseño innovadoras que permitan la concepción y diseño preliminar de las propiedades estructurales de estructuras que sean capaces de controlar sus demandas de aceleración dentro de umbrales aceptables de acuerdo al daño permisible en los contenidos.

En términos de sistemas estructurales apoyados sobre bases rígidas, se ha observado que su incursión al rango plástico de comportamiento o el uso de amortiguamiento adicional suele reducir las demandas de aceleración (Adam et al. 2003, Ahmadi y Su 1992, Chaudhuri y Hutchinson 2004, Fan y Ahmadi 1992, Retamales et al. 2006, Villaverde 2006). Aunque esto representa una posibilidad en términos de controlar dichas demandas, lo cierto es que el comportamiento plástico representa daño estructural, y que las formulaciones analíticas requeridas para estimar las demandas de aceleración resultan muy complejas. Una alternativa para el control de daño en contenidos es el uso de aislamiento sísmico. Múltiples estudios experimentales y analíticos han demostrado que el aislamiento de base es por mucho la mejor forma de controlar las demandas de aceleración en una super-estructura (Clark et al. 2002, Kelly y Tsai 1985). Sin embargo, es necesario considerar que no todos los sistemas de aislamiento representan una opción atractiva en estos términos (Ahmadi y Su 1992); y que a pesar de su potencial en términos de controlar las demandas de aceleración, la concepción y diseño de sistemas estructurales con aislamiento de base suelen utilizar formatos de diseño basados en resistencia y desplazamiento (Naeim y Kelly 1999, Priestley et al. 2007). Lo anterior resulta en que el control de las demandas de aceleración no sea contemplado desde el inicio, y que dichas demandas deben ser controladas a partir de un procedimiento iterativo que involucra múltiples análisis paso a paso.

Este artículo analiza el uso de sistemas de aislamiento de base para controlar las demandas de aceleración de entrepiso de sistemas estructurales de baja altura. Se estudia el efecto que en dichas demandas tienen la rigidez lateral y capacidad de disipación de energía del sistema de aislamiento, y la rigidez lateral de la super-estructura. Mediante la integración de los resultados presentados, se discute el uso de un modelo equivalente de un grado de libertad para la concepción y diseño basada en aceleración de las propiedades estructurales del sistema aislado. El estudio se limita a super-estructuras de baja altura cuyo sistema de aislamiento exhibe comportamiento lineal y amortiguamiento de tipo viscoso. Se consideran movimientos del terreno registrados en sitios de suelo firme ubicados en la costa del Pacífico Mexicano. La diferencia entre los niveles de amortiguamiento de la super-estructura y su sistema de aislamiento es considerada a través del uso del enfoque de amortiguamiento no-clásico.

\section{PROPIEDADES DINÁMICAS DE SISTEMAS AISLADOS}

En sistemas estructurales donde existen diferencias sustanciales de amortiguamiento entre las partes que los componen, no es posible emplear la teoría del amortiguamiento clásico. Debido a lo anterior, es necesario el uso de un enfoque que considere el efecto de la diferencia de amortiguamientos, denominado enfoque de amortiguamiento no-clásico (Hurty, 1965). La ecuación de movimiento para sistemas estructurales elásticos con aislamiento sísmico de base (como el mostrado en la Figura 1) puede plantearse como (Naeim y Kelly, 1999): 
$M^{*} \ddot{v}^{*}+C^{*} \ddot{v}^{*}+K^{*} v^{*}=-M^{*} r^{*} \ddot{u}_{g}$

donde $M^{*}, C^{*}$ y $K^{*}$ son las matrices de masa, amortiguamiento y rigidez del sistema aislado, respectivamente; $v^{*}$ es el vector de desplazamientos relativos al sistema de aislamiento; $r^{*}$ es un vector de acoplamiento que relaciona los grados del sistema estructural con la aceleración del terreno; y ü $\ddot{g}_{g}$ es la aceleración del terreno.

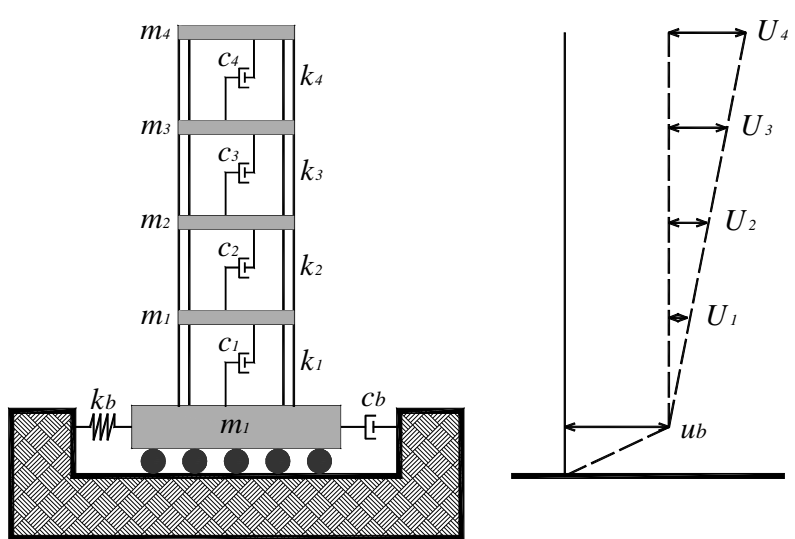

Figura 1. Modelo idealizado para sistema aislado de cuatro niveles (cinco grados de libertad)

La ecuación de movimiento mostrada en la ecuación 1 puede expresarse en forma matricial extendida para un sistema de $N$ grados de libertad como:

$\left[\begin{array}{cc}m+m_{b} & r^{T} M \\ M r & M\end{array}\right]\left\{\begin{array}{l}\ddot{u}_{b} \\ \ddot{U}\end{array}\right\}+\left[\begin{array}{cc}c_{b} & 0 \\ 0 & C\end{array}\right]\left\{\begin{array}{l}\dot{u}_{b} \\ \dot{U}\end{array}\right\}+\left[\begin{array}{cc}k_{b} & 0 \\ 0 & K\end{array}\right]\left\{\begin{array}{l}u_{b} \\ U\end{array}\right\}=-\left[\begin{array}{cc}m+m_{b} & r^{T} M \\ M r & M\end{array}\right]\left\{\begin{array}{l}1 \\ 0\end{array}\right\} \ddot{u}_{g}$

donde $m$ es la masa total de la super-estructura; $m_{b}, c_{b}$ y $k_{b}$ son la masa, el amortiguamiento y la rigidez del sistema de aislamiento, respectivamente; $M, C$ y $K$ son las matrices de masas, amortiguamiento y rigidez, respectivamente, de la super-estructura sobre base rígida; $r$ es un vector de unos; $u_{b}$ es el desplazamiento medido en el nivel de aislamiento; y $U$ es el vector de desplazamientos de la super-estructura medidos respecto al desplazamiento del sistema de aislamiento. El sistema matricial resumido en la ecuación 2 contempla $N$ ecuaciones.

Para el caso de sistemas en que el amortiguamiento no pueda ser tratado de forma clásica, las formas modales, frecuencias y porcentajes de amortiguamiento modal dependen de la matriz de amortiguamiento. En este caso, la ecuación de movimiento se transforma en una ecuación diferencial de primer orden, lo que simplifica considerablemente la solución del problema. Para esto, los desplazamientos del sistema se representan conforme a lo siguiente:

$Z=\left\{\begin{array}{l}\dot{v}^{*} \\ v^{*}\end{array}\right\}$

Al emplear la variable $z$, que representa los desplazamientos del sistema, es posible transformar la ecuación 1 en la forma de representación de estados: 
$\left[\begin{array}{cc}{[0]} & M^{*} \\ M^{*} & C^{*}\end{array}\right] \dot{z}+\left[\begin{array}{cc}-M^{*} & {[0]} \\ {[0]} & K^{*}\end{array}\right] Z=-\left\{\begin{array}{c}\{0\} \\ M^{*}\{1\}\end{array}\right\} \ddot{u}_{g}$

Si se definen las matrices $[A]$ y $[B]$ como:

$$
\begin{aligned}
& {[A]=\left[\begin{array}{ll}
{[0]} & M^{*} \\
M^{*} & C^{*}
\end{array}\right]} \\
& {[B]=\left[\begin{array}{cc}
-M^{*} & {[0]} \\
{[0]} & K^{*}
\end{array}\right]}
\end{aligned}
$$

Es posible reescribir la ecuación 4 como:

$$
[A] \dot{z}+[B] z=0
$$

Para sistemas dinámicos invariantes en el tiempo y sometidos a vibración libre, la solución en términos del desplazamiento y velocidad del sistema puede expresarse como:

$$
\begin{aligned}
& z=y e^{p t} \\
& \dot{z}=p y e^{p t}
\end{aligned}
$$

Al sustituir las ecuaciones 8 y 9 en la ecuación 7, se llega a la solución estándar de eigenvalores para un sistema dinámico:

$$
\left[[B]^{-1}[A] p-[I] y\right]=0
$$

Los valores de $p$ y $y$, que representan los valores y vectores característicos del problema, permiten establecer las frecuencias, porcentajes de amortiguamiento modal y las formas modales del sistema estructural. La ecuación 10 contempla un sistema de $2 N$ ecuaciones. La estimación de los valores y vectores característicos puede plantearse a partir de:

$$
\begin{aligned}
& p=\frac{1}{\lambda} \\
& y=\left\{\begin{array}{c}
p \phi \\
\phi
\end{array}\right\}
\end{aligned}
$$

Cabe destacar que la solución queda expresada en términos de números complejos. En el caso del valor inverso de $p$, se definen la parte real e imaginaria y su significado en la solución del sistema: 
$\lambda_{j}=-\omega_{n j} \zeta_{j}+i \omega_{d j}$

$\left|\lambda_{j}\right|=\omega_{n j}$

$\omega_{d j}=\omega_{n j} \sqrt{\left|1-\zeta_{j}^{2}\right|}$

donde $\omega_{n j}$ es la frecuencia natural no amortiguada asociada al modo $j$; $\omega_{d j}$ es la frecuencia amortiguada del modo $j$; y $\zeta_{j}$ es el porcentaje de amortiguamiento modal asociado al modo $j$.

Una vez obtenidas las propiedades dinámicas del sistema estructural con amortiguamiento no-clásico, resulta importante conocer la participación de cada uno de sus modos de vibrar en su respuesta dinámica total (Hurty, 1965):

$\Gamma_{j}=2 \operatorname{Im}\left(p_{j}\right)\left|\frac{\phi M^{*}\{1\}}{y_{j}^{T}[A] y_{j}}\right|$

donde $\Gamma_{j}$ es el factor de participación modal asociado al modo $j$; $\operatorname{Im}\left(p_{j}\right)$ es la parte imaginaria de $p_{j}$; y el resto de los parámetros han sido definidos previamente. Es importante hacer notar que aunque diferente desde un punto de vista matemático, la respuesta de un sistema no clásico puede ser explicado por medio de la misma base conceptual utilizada para los sistemas clásicos (esto es: frecuencias, modos de vibrar, factores de participación modal, etc.).

Otro aspecto relevante para la respuesta dinámica de estructuras aisladas es la relación que existe entre las masas de la super-estructura y del nivel de aislamiento. La relación de masas $(\gamma)$ puede ser expresada conforme a (Naeim y Kelly, 1999):

$\gamma=\frac{\sum_{i=1}^{N-1} m_{i}}{m_{b}+\sum_{i=1}^{N-1} m_{i}}$

donde $m_{i}$ es la masa en cada grado de libertad de la super-estructura; y $m_{b}$ la masa en el nivel de aislamiento.

\section{Factores de participación modal}

De acuerdo a la teoría lineal, el modo fundamental de vibración de una estructura aislada está relacionado cercanamente al grado de libertad asignado al desplazamiento lateral del sistema de aislamiento. En este sentido, son los modos superiores de vibrar los que contribuyen a la deformación lateral y movimiento de la super-estructura, y por tanto, al daño en los sistemas estructural, no estructural y en los contenidos de la estructura aislada (Naeim y Kelly, 1999). Dentro de este contexto, es de interés estudiar la variación del factor de participación modal asociado al modo fundamental de vibración de varios sistemas aislados para entender cómo controlar los efectos de modos superiores. Lo anterior permite visualizar las propiedades de los sistemas aislados que deben tomarse en cuenta, y en que rangos de valores, para controlar las demandas de aceleración en un estructura aislada. Para darle un carácter 
absoluto al valor de los factores de participación modal, en este trabajo dichos factores se han normalizado de tal forma que su sumatoria sea igual a uno, de tal manera que, por ejemplo, un factor de participación modal para el modo fundamental de vibración $\left(\Gamma_{1}\right)$ igual que uno indica que la respuesta lateral del sistema estructural está totalmente dominada por dicho modo. En términos de porcentaje, un $\Gamma_{1}$ de $100 \%$ implica una respuesta lateral totalmente dominada por el modo fundamental de vibración.

Este artículo contempla super-estructuras elásticas que consisten en una serie de masas (una por piso), conectadas a través de resortes elásticos que condensan la rigidez lateral de los diferentes entrepisos. El valor de la rigidez lateral, amortiguamiento y masa se consideró uniforme en toda la altura de la superestructura. En cuanto al comportamiento lateral de los sistemas estructurales, se supuso que está dominado por deformaciones globales a corte. En cuanto al sistema de aislamiento, se consideró un comportamiento elástico con amortiguamiento viscoso. Este comportamiento es típico de aisladores de caucho con amortiguamiento normal o alto, o el de aisladores de caucho complementados con amortiguadores viscosos. Debe mencionarse que las herramientas actuales de análisis proveen un modelado razonable de la respuesta dinámica global y local de estructuras aisladas reales (Kelly 1982, Ventura et al. 2003).

Una medida que caracteriza a los sistemas estructurales en el presente trabajo es la relación de rigideces laterales que exhiben el sistema de aislamiento y su super-estructura $\left(T_{b} / T_{s}\right)$, donde $T_{b}$ es el periodo fundamental que exhibiría el sistema aislado si toda su masa se concentrará en la base, y $T_{s}$ es el periodo fundamental de la super-estructura sobre base rígida. La tabla 1 resume las propiedades estructurales y dinámicas de los sistemas de dos, cuatro y ocho niveles bajo consideración. $\zeta_{b}$ y $\zeta_{s}$ son los porcentajes de amortiguamiento crítico asignados al sistema de aislamiento y a la super-estructura, respectivamente. Es importante destacar que aunque las super-estructuras de ocho niveles salen del alcance planteado para este artículo (estructuras de baja altura), se incluyen sus resultados para aportar al lector un panorama más amplio a partir del cual le sea posible entender las particularidades que se dan alrededor de las estructuras aisladas de baja altura. En este sentido, los resultados de dos y ocho niveles aquí reportados sólo representan una medida de comparación con respecto a las estructuras de interés (cuatro niveles).

Tabla 1. Propiedades de los sistemas estructurales bajo consideración

\begin{tabular}{|c|c|c|c|c|}
\hline Sistema & $T_{b}$ (seg) & $\zeta_{b}(\%)$ & $T_{s}$ (seg) & $\zeta_{s}(\%)$ \\
\hline \multirow{4}{*}{2 Niveles } & \multirow{12}{*}{$\begin{array}{l}1.5 \\
2.0 \\
2.5 \\
3.0\end{array}$} & \multirow{12}{*}{$\begin{array}{l}10 \\
15 \\
20 \\
25\end{array}$} & 0.05 & \multirow{12}{*}{2} \\
\hline & & & 0.17 & \\
\hline & & & 0.28 & \\
\hline & & & 0.40 & \\
\hline \multirow{4}{*}{4 Niveles } & & & 0.15 & \\
\hline & & & 0.30 & \\
\hline & & & 0.50 & \\
\hline & & & 0.70 & \\
\hline \multirow{4}{*}{8 Niveles } & & & 0.30 & \\
\hline & & & 0.50 & \\
\hline & & & 0.70 & \\
\hline & & & 1.00 & \\
\hline
\end{tabular}

Para el caso de una super-estructura de dos niveles, en la figura 2 se indica el factor de participación asociado al primer modo de vibrar $\left(\Gamma_{1}\right)$ para distintos valores de $\gamma \mathrm{y} \zeta_{b}$. La figura muestra que en estructuras aisladas de pocos niveles, el nivel de amortiguamiento en el sistema de aislamiento tiene poca influencia sobre la participación del primer modo de vibrar. Sin embargo y conforme a lo mostrado, la relación de masas puede afectar levemente la participación del primer modo de vibrar en la respuesta dinámica de la estructura. 
Con un incremento de $\gamma$ (la masa en la super-estructura se incrementa en relación con la del sistema de aislamiento), el factor de participación tiende a incrementarse. Conforme se incrementa el valor de $T_{b} / T_{s}$ de cero a 10, $\Gamma_{1}$ se incrementa rápidamente. Una vez que $T_{b} / T_{s}$ alcanza el valor de diez, $\Gamma_{1}$ tiende a incrementarse con una tasa menor; y a una todavía menor una vez que $T_{b} / T_{s}$ excede el valor de 16 . Esto implica, en congruencia con la teoría lineal discutida por Naeim y Kelly (1999), que un incremento en $T_{b} / T_{s}$ resulta, para valores pequeños y moderados de este cociente, en una reducción muy importante de la participación de los modos superiores en la deformación lateral y respuesta dinámica de la super-estructura.

Las Figuras 3 y 4, respectivamente, muestran valores de $\Gamma_{1}$ para sistemas aislados de cuatro y ocho pisos. Contrario a lo que se muestra en la figura $2, \zeta_{b}$ exhibe una influencia moderada en $\Gamma_{1}$, particularmente en los casos en que $T_{b} / T_{s}$ es menor que ocho. Mientras que $\gamma$ sigue exhibiendo una influencia moderada en el valor de $\Gamma_{1}$, es posible observar que un incremento en $\gamma$ resulta en factores de participación mayores, excepto para valores muy pequeños de $T_{b} / T_{s}$.
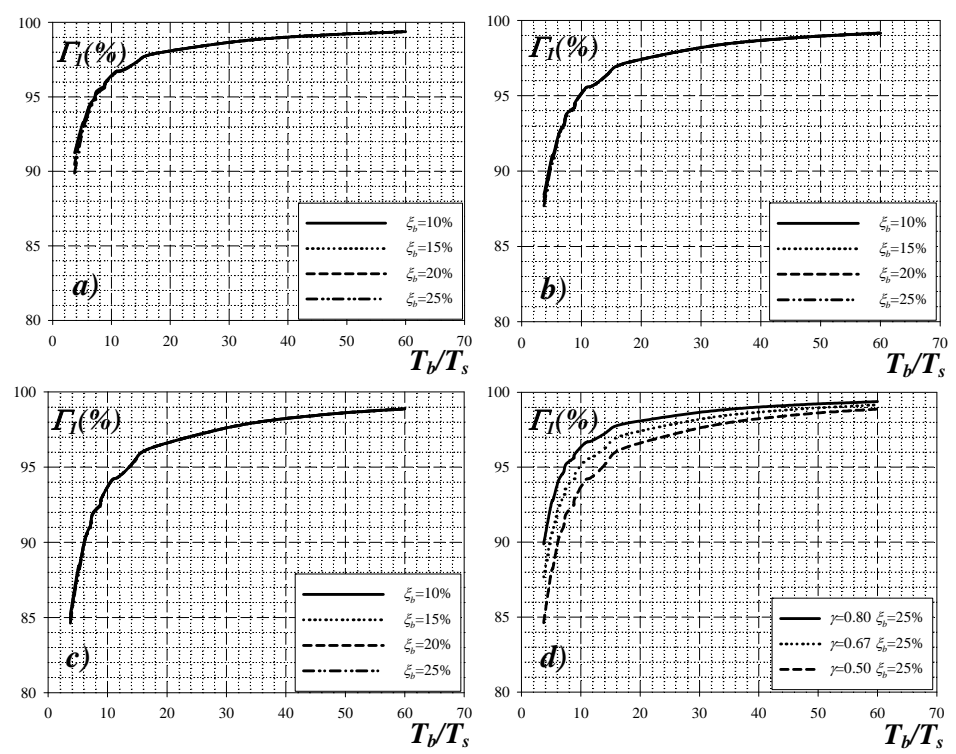

Figura 2. Factor de participación modal asociado al primer modo de vibrar de super-estructuras aisladas de dos pisos: a) $\gamma=0.80$; b) $\gamma=0.67$; c) $\gamma=0.50$; d) $\zeta_{b}=0.25$

\section{RESPUESTA DE SISTEMAS ESTRUCTURALES AISLADOS}

En términos de control de aceleración, es conveniente estudiar la respuesta dinámica de sistemas aislados sujetos a la acción de sismos intensos. Con este propósito se utilizaron un conjunto de veintidós movimientos del terreno registrados en zonas de suelo firme ubicadas en la costa del Pacífico Mexicano. La amplitud de dichos movimientos se escaló de tal forma que exhibieran la aceleración máxima del terreno correspondiente a un periodo de retorno de quinientos años para la costa del estado de Guerrero. En términos generales, puede decirse que el conjunto de registros representa el sismo de diseño para estructuras construidas en terrenos firmes localizados a lo largo de la costa de Guerrero. La figura 5 muestra la media de los espectros elásticos de pseudo-aceleración $\left(S_{d} / g\right)$ y desplazamiento $\left(S_{d}\right)$ para diferentes porcentajes de amortiguamiento crítico (§). Note que las demandas de desplazamiento son moderadas para periodos mayores que dos segundos, de tal forma que a diferencia de lo observado en las zonas epicentrales de lugares como 
California y Japón, la demanda de desplazamiento en estructuras aisladas en la costa del Pacífico Mexicano no representa una limitación para su concepción basada en aceleración.
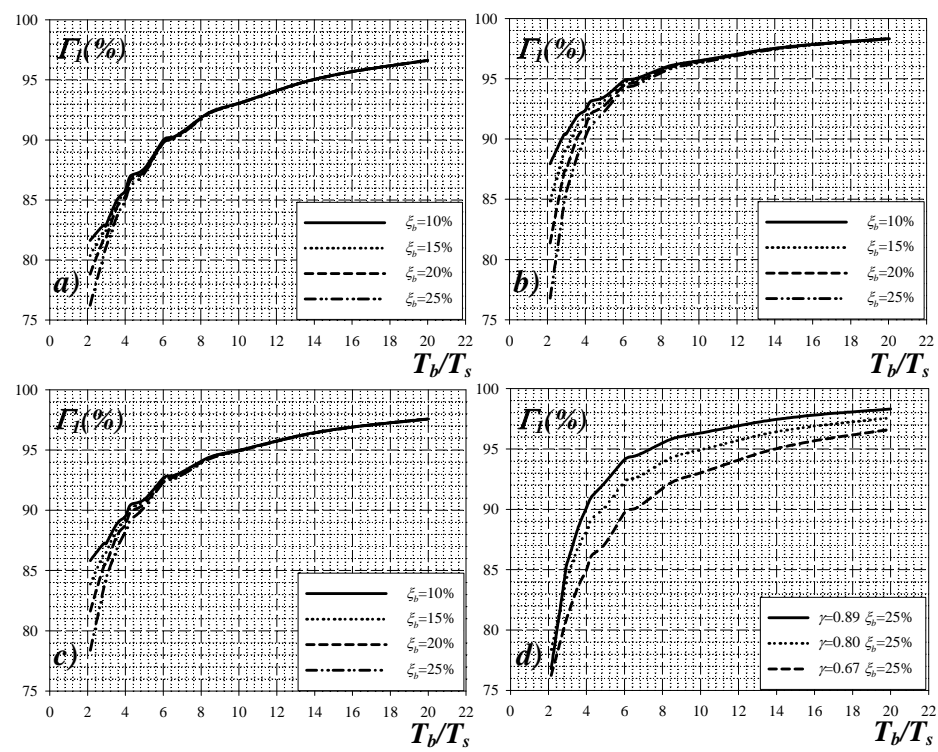

Figura 3. Factor de participación modal asociado al primer modo de vibrar de super-estructuras aisladas de cuatro pisos: a) $\gamma=0.89$; b) $\gamma=0.80$; c) $\gamma=0.67$; d) $\zeta_{b}=0.25$
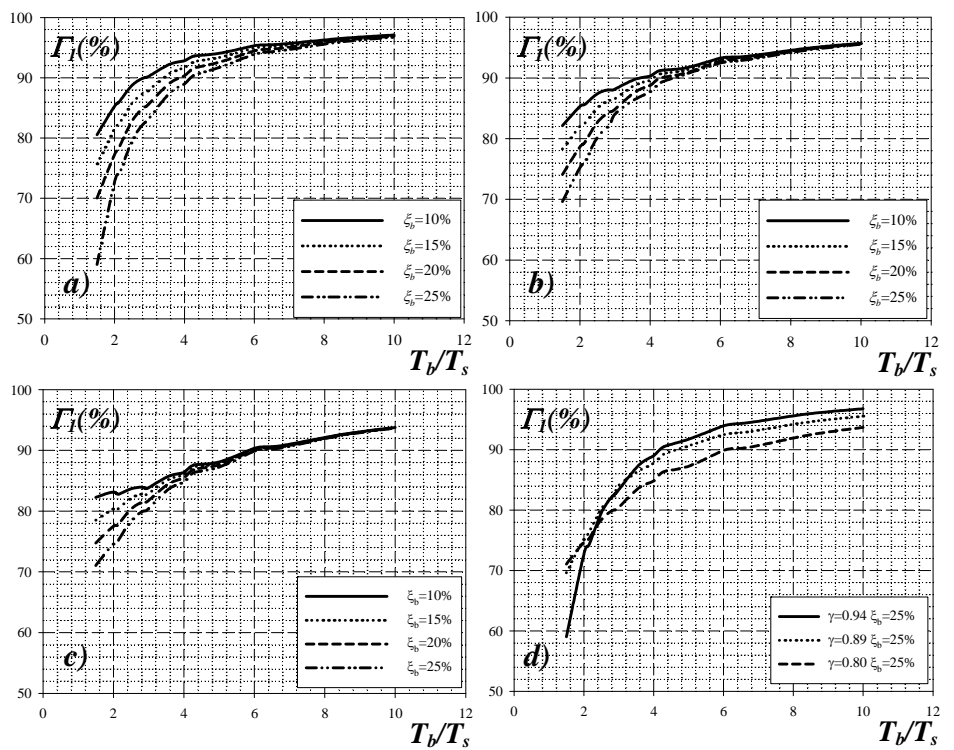

Figura 4. Factor de participación modal asociado al primer modo de vibrar de super-estructuras aisladas de ocho pisos: a) $\gamma=0.94$; b) $\gamma=0.89$; c) $\gamma=0.80$; d) $\zeta_{b}=0.25$

El movimiento lateral que exhibe un sistema estructural aislado puede utilizarse para identificar la participación que en su respuesta dinámica tienen sus diferentes modos de vibrar. Esto puede lograrse a través de dos representaciones: A) En el dominio de la frecuencia a través de espectros de Fourier; y B) En el dominio del tiempo a través de espectros de piso. 


\section{Dominio de la frecuencia}

La amplitud de los espectros de Fourier proporciona información directa sobre el contenido de frecuencias de la respuesta de entrepiso, y en este sentido, permite el entendimiento de como el movimiento es filtrado por la estructura aislada desde el nivel del terreno hasta los niveles superiores. Las figuras 6 y 7 muestran espectros de Fourier obtenidos a partir de la historia de aceleración absoluta de azotea de cada uno de los 22 registros utilizados en el estudio. Los valores corresponden a estructuras aisladas de cuatro niveles. Mientras que la rigidez lateral y el nivel de amortiguamiento del sistema de aislamiento varían, se considera un valor de $\gamma$ de 0.8 para todos los sistemas. La super-estructura analizada en la figura 6 puede considerarse flexible ( $T_{s}=0.7$ segundos para una estructura de cuatro pisos cimentada sobre base rígida); y la contemplada en la figura 7 , como rígida $\left(T_{s}=0.15\right.$ segundos)
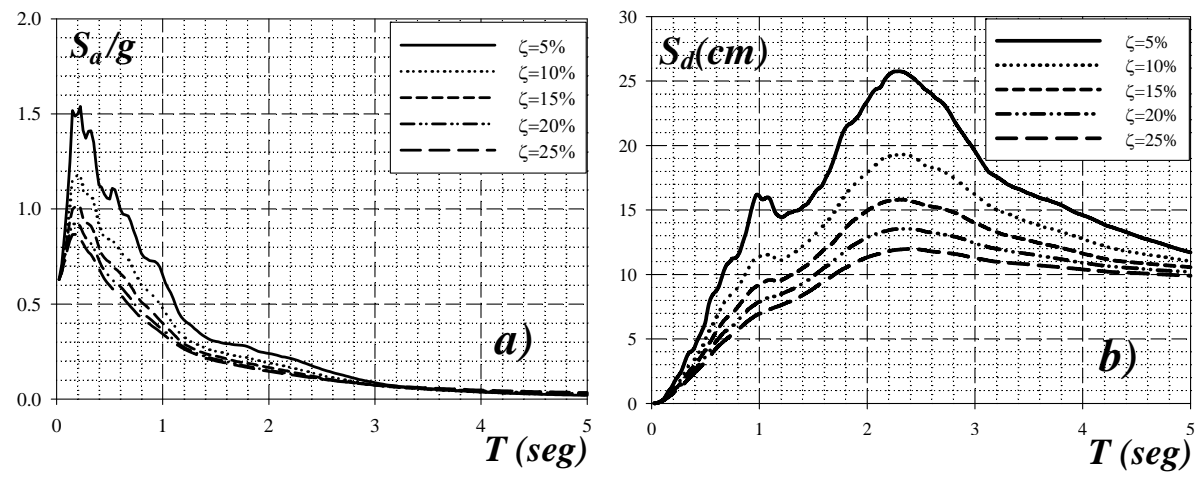

Figura 5. Espectros elásticos promedio para movimientos del terreno bajo consideración:

a) Seudo-aceleración; b) Seudo-desplazamiento

Los espectros de Fourier mostrados en las Figuras 6 y 7 exhiben un gran pico en la banda de frecuencias localizada alrededor de la frecuencia fundamental de vibración del sistema aislado. En el caso particular de la figura 6, los espectros exhiben picos notables de respuesta en la zona de frecuencias altas $(f>$ $2 \mathrm{~Hz}$ ), los cuales están asociados a los modos superiores del sistema aislado. Mientras que la ubicación de los picos menores es sensiblemente independiente de $\zeta_{b}$, su amplitud se incrementa en términos relativos con un incremento de $\zeta_{b}$. Lo mostrado en la figura 6 es congruente con los factores de participación resumidos en la figura 3. Particularmente, un incremento en el nivel de amortiguamiento del sistema de aislamiento se refleja en una mayor contribución de los modos superiores. La comparación de los resultados mostrados en las figuras 6 y 7 permite entender la influencia de $T_{b} / T_{s}$ en la respuesta dinámica de los sistemas aislados. El resultado final de incrementar el valor de $T_{b} / T_{s}$ es la desaparición de los picos menores localizados en frecuencias altas.

\section{Dominio en el tiempo}

Aunque no siempre es posible utilizar un espectro de piso para evaluar las demandas de aceleración en los contenidos, esta representación es muy útil para entender cuáles son las acciones que deben tomarse en cuenta, desde un punto de vista estructural, para controlar las demandas de aceleración en un sistema aislado. Es importante mencionar que los espectros de piso no pueden ser aplicados para evaluar el desempeño de aquellos contenidos que son capaces, debido a su masa y propiedades dinámicas, de interactuar dinámicamente con la super-estructura a tal grado de modificar su respuesta global. Bajo este contexto, se ha observado que los espectros de piso proporcionan estimaciones razonables de las demandas de aceleración en contenidos cuyas masas son mucho más pequeñas que las de la estructura en la que se alojan, y cuyas frecuencias no se acercan a una de las frecuencias naturales del sistema estructural (Villaverde, 1997, 2006). 
En el caso de contenidos resonantes, los espectros de piso desprecian la interacción dinámica e ignoran el hecho de que las componentes de movimiento de sistemas con diferente amortiguamiento pueden darse fuera de fase, lo que resulta en demandas de aceleración conservadoras.
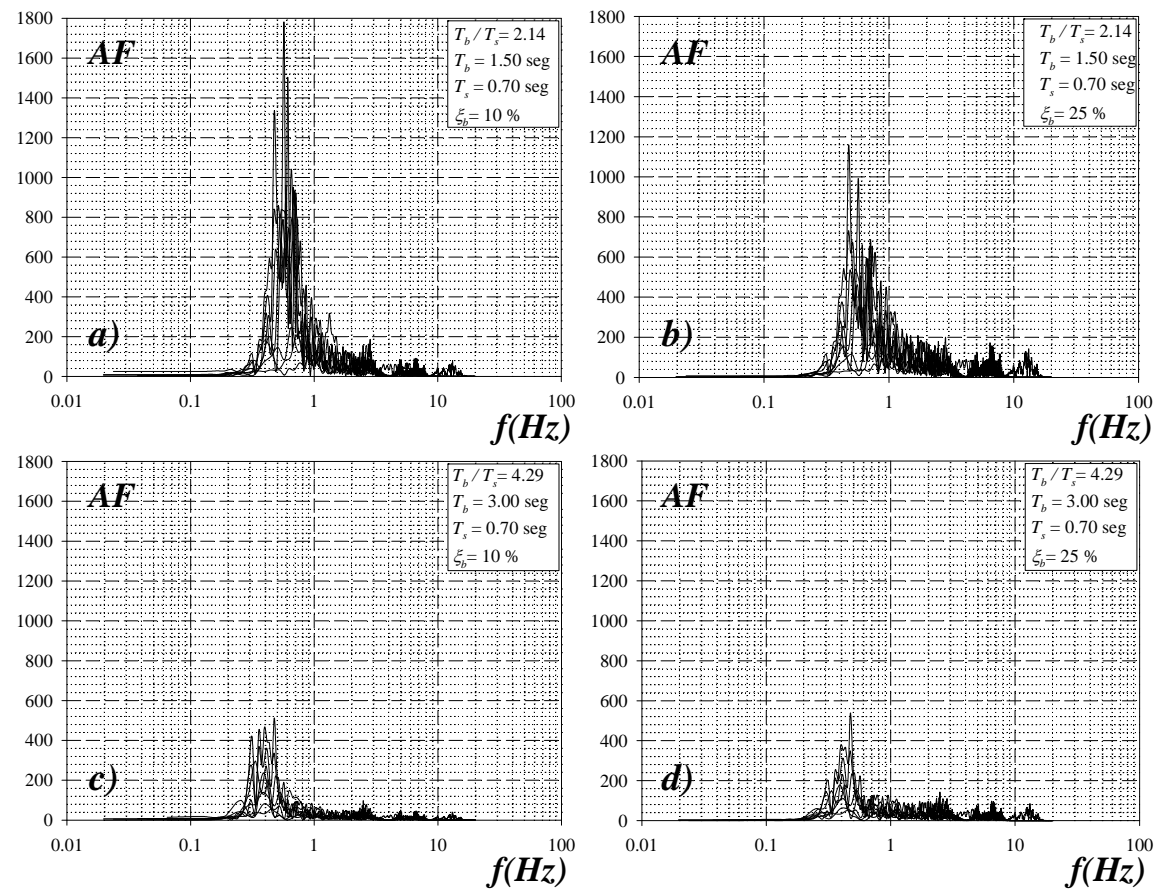

Figura 6. Espectros de Fourier correspondientes a la historia de aceleración absoluta de azotea de superestructura flexible de cuatro niveles
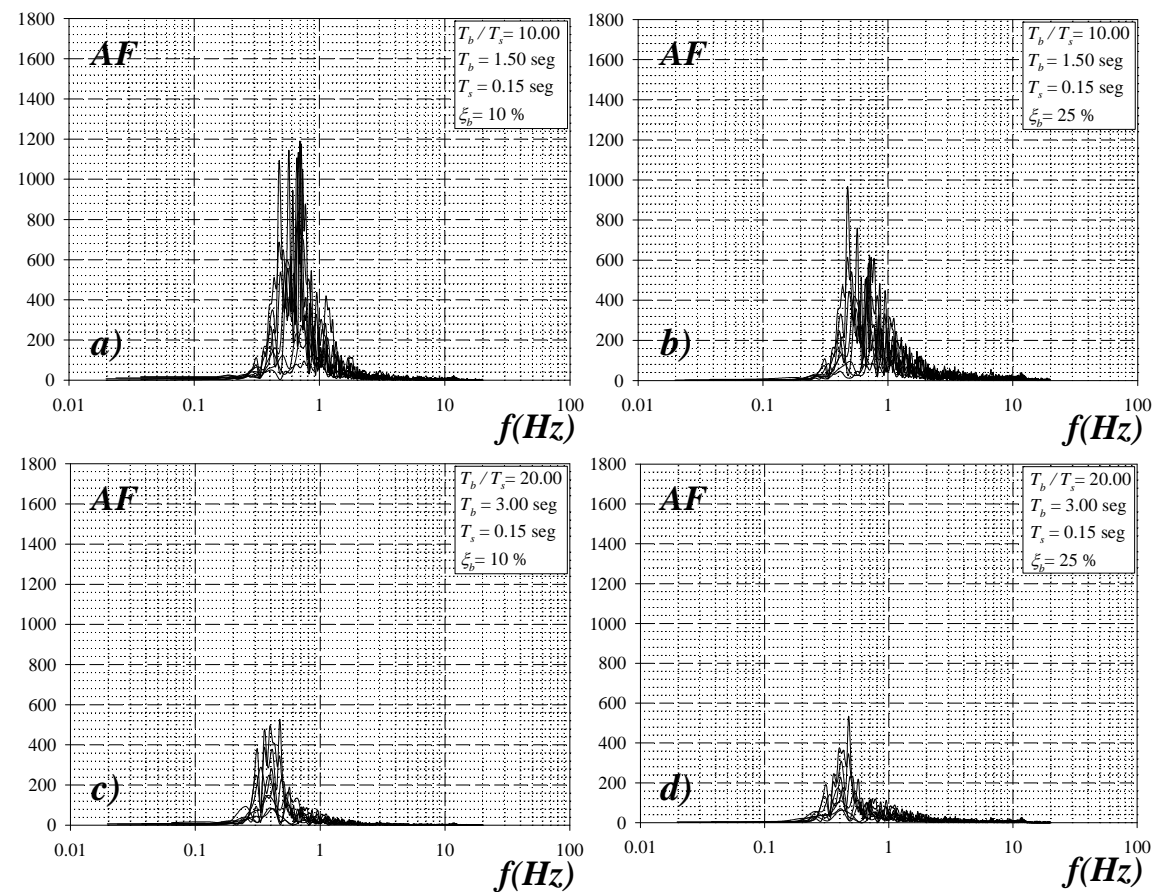

Figura 7. Espectros de Fourier correspondientes a la historia de aceleración absoluta de azotea de superestructura rígida de cuatro niveles 
De acuerdo a la observación hecha por Sankaranarayanan (2007) y Sankaranarayanan y Medina (2007), pueden considerarse tres regiones de periodo para un espectro de piso: A) Cortos; B) Intermedios; y C) Largos. La primera región contempla contenidos con periodos tales que la relación entre su periodo $\left(T_{e}\right)$ y $T_{b}$ no exceden de 0.5 . La relación de periodos intermedios va de 0.5 a 1.5 , y la correspondiente a periodos largos excede el valor de 1.5 .

Las figuras 8 y 9 muestran espectros de piso de aceleración absoluta y velocidad para la azotea de estructuras aisladas de cuatro niveles. Los espectros, obtenidos para contenidos con $2 \%$ de amortiguamiento crítico, representan la media espectral de aquellos correspondientes a los registros bajo consideración. Debe notarse que las figuras contemplan diferentes valores de $\zeta_{b}$ y $T_{b} / T_{s}$.

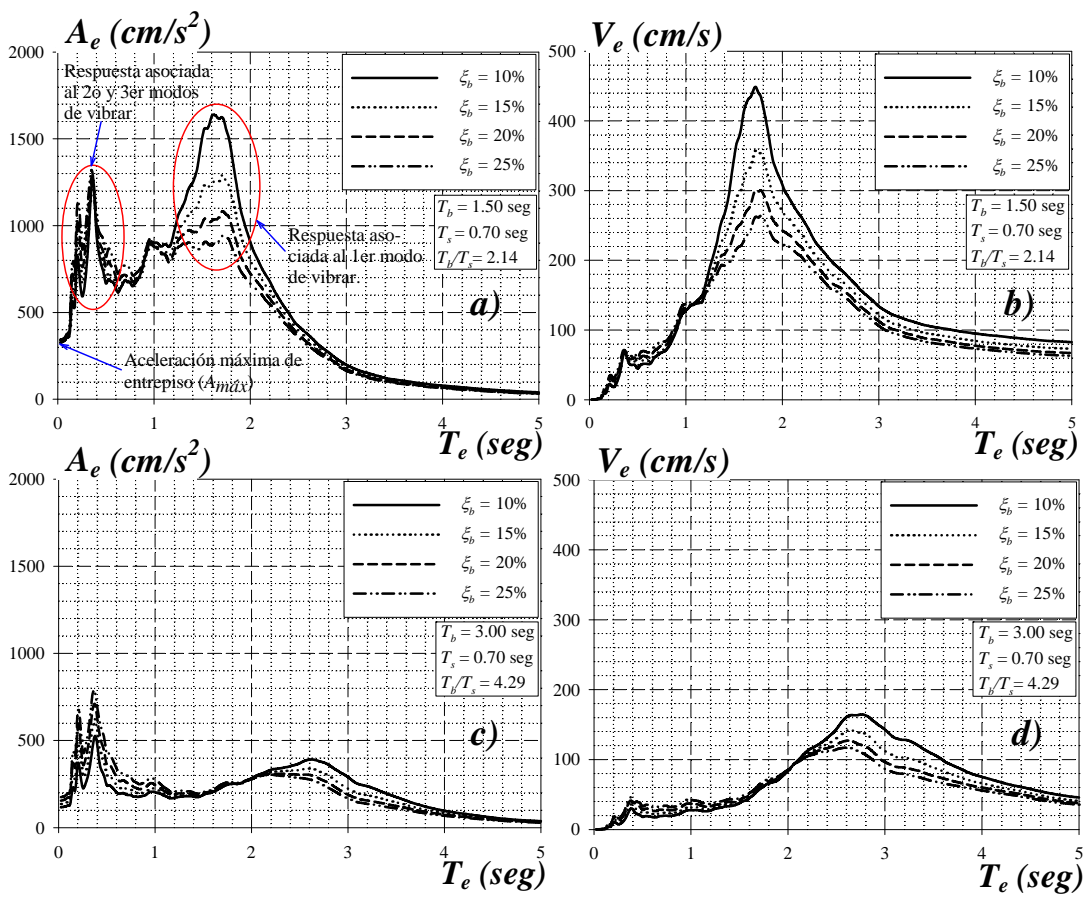

Figura 8. Espectros de aceleración absoluta y velocidad de azotea correspondientes a super-estructura flexible de cuatro pisos

Debido a la participación de modos superiores, los espectros de aceleración incluidos en la figura 8 para una super-estructura flexible exhiben amplificaciones considerables de aceleración en el rango de periodos cortos. Particularmente, hay dos picos notables asociados al segundo y tercer modo de vibrar. Aunque la participación de estos dos modos también es notoria en términos de los espectros de velocidad, su influencia no resulta en incrementos sustanciales en las demandas de velocidad para el rango de periodos cortos. En este sentido, el control de la velocidad no parece ser tan importante como lo es el control de la aceleración para un desempeño sísmico adecuado de contenidos que caigan en la zona de periodos cortos. Los resultados mostrados en la figura 9 ayudan a entender las consecuencias de incrementar la rigidez lateral de la super-estructura. En términos generales y en congruencia con la teoría lineal discutida por Naeim y Kelly (1999), puede decirse que la contribución de los modos superiores a las demandas de aceleración de los contenidos disminuye considerablemente. Los resultados resumidos en las figuras 8 y 9 enfatizan una vez más que en términos del control de los modos superiores, la situación ideal para un sistema aislado puede formularse en términos de un sistema de aislamiento flexible con una super-estructura muy rígida. Debe notarse que aunque el incremento de $\zeta_{b}$ reduce la respuesta de los contenidos que caen en la región intermedia 
de periodos; la mayoría de los contenidos reales tienen periodos que caen en el rango de periodos cortos y que, en este rango, el incremento de $\zeta_{b}$ no se traduce en menores demandas de aceleración. La última observación coincide con lo observado para las demandas de aceleración en sistemas con base rígida en términos de la poca efectividad que tiene la disipación de energía para reducir la contribución de la respuesta de modos superiores (Rodríguez et al. 2007, Dowell et al. 2008, Clayton y Medina 2010). Es importante hacer notar que el incremento en la rigidez lateral de la super-estructura, relativa a la del sistema de aislamiento, permite que un sistema complejo de múltiples grados de libertad (como los descritos por Medina et al. 2006, Sankaranarayanan y Medina 2007, Clayton y Medina 2010, y Dowell et al. 2008) exhiba un comportamiento similar al de un sistema de un grado de libertad con un solo pico de respuesta en el espectro de piso (como el descrito por Lin y Mahin 1985, Igusa 1990).
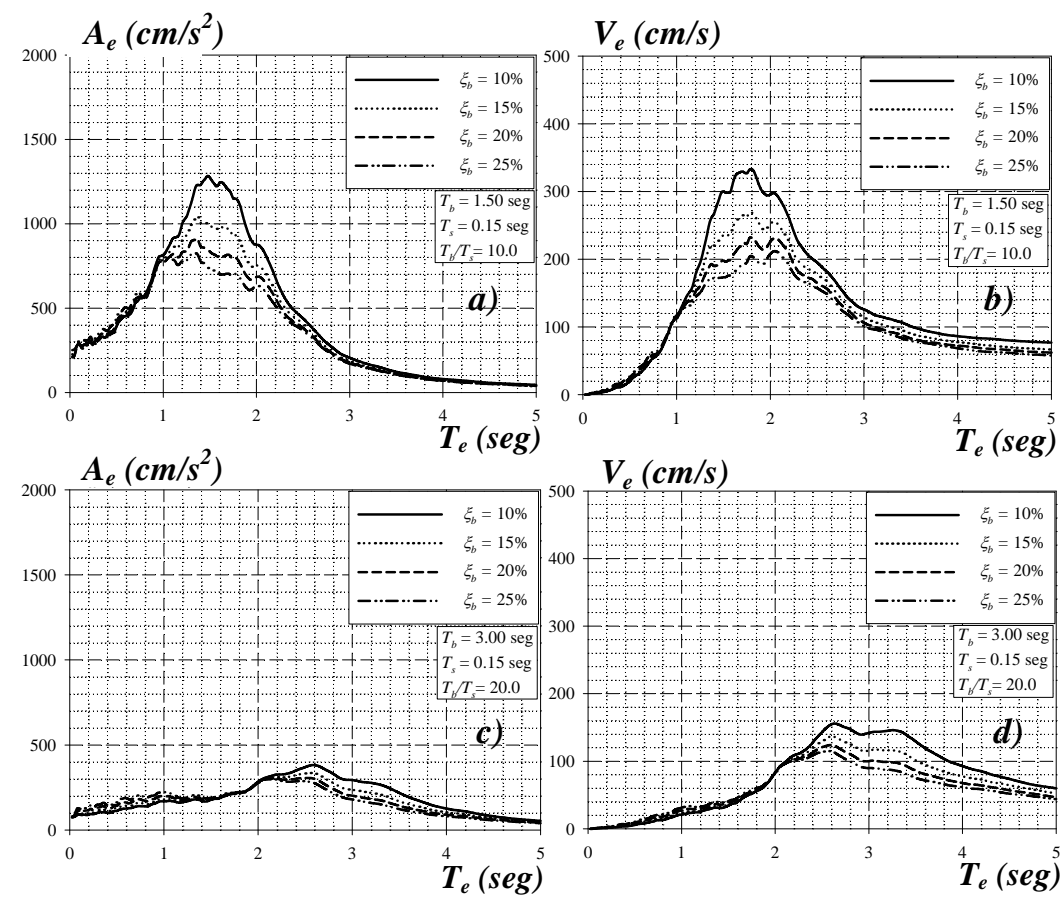

Figura 9. Espectros de aceleración absoluta y velocidad de azotea correspondientes a super-estructura rígida de cuatro pisos

\section{RELACIONES DE MÁXIMA ACELERACIÓN DE AZOTEA RESPECTO A LA MÁXIMA ACELERACIÓN EN EL SISTEMA DE AISLAMIENTO}

Otra manera de caracterizar la amplificación de aceleración en un sistema aislado es a través de un cociente de aceleraciones:

$$
R_{N P}=\frac{\max \left(\ddot{u}_{n}\right)}{\max \left(\ddot{u}_{b}\right)}
$$

donde $n$ es el número de pisos de la super-estructura; $\ddot{u}_{n}$ representa el registro de aceleración absoluta en el nivel de azotea; y $\ddot{u}_{B}$ representa el registro de aceleración absoluta en el nivel de aislamiento (base).

La figura 10a muestra cocientes de aceleración para una estructura aislada de cuatro niveles con $\zeta_{b}$ de $10 \%$ y $\gamma$ de 0.80 . Se observa una reducción importante en la dispersión de $R_{4}$ con un incremento de $T_{b} / T_{s}$. 
Puede decirse que no sólo el nivel de amplificación del movimiento decrece en la super-estructura conforme su rigidez lateral se incrementa, sino que la reducción de los efectos de los modos superiores se refleja en una mayor certidumbre en términos de la predicción de los niveles de aceleración. En las figuras 10b a 10d se muestran tendencias centrales para los valores de $R_{4}$. Conforme el valor de $\gamma$ se incrementa, el valor de $R_{4}$ tiende a disminuir para un valor dado de $T_{b} / T_{s}$, particularmente para valores pequeños de la relación de periodos. Un incremento en $\zeta_{b}$ resulta en ligeros incrementos de $R_{4}$, independientemente de la relación de $T_{b} / T_{s}$.
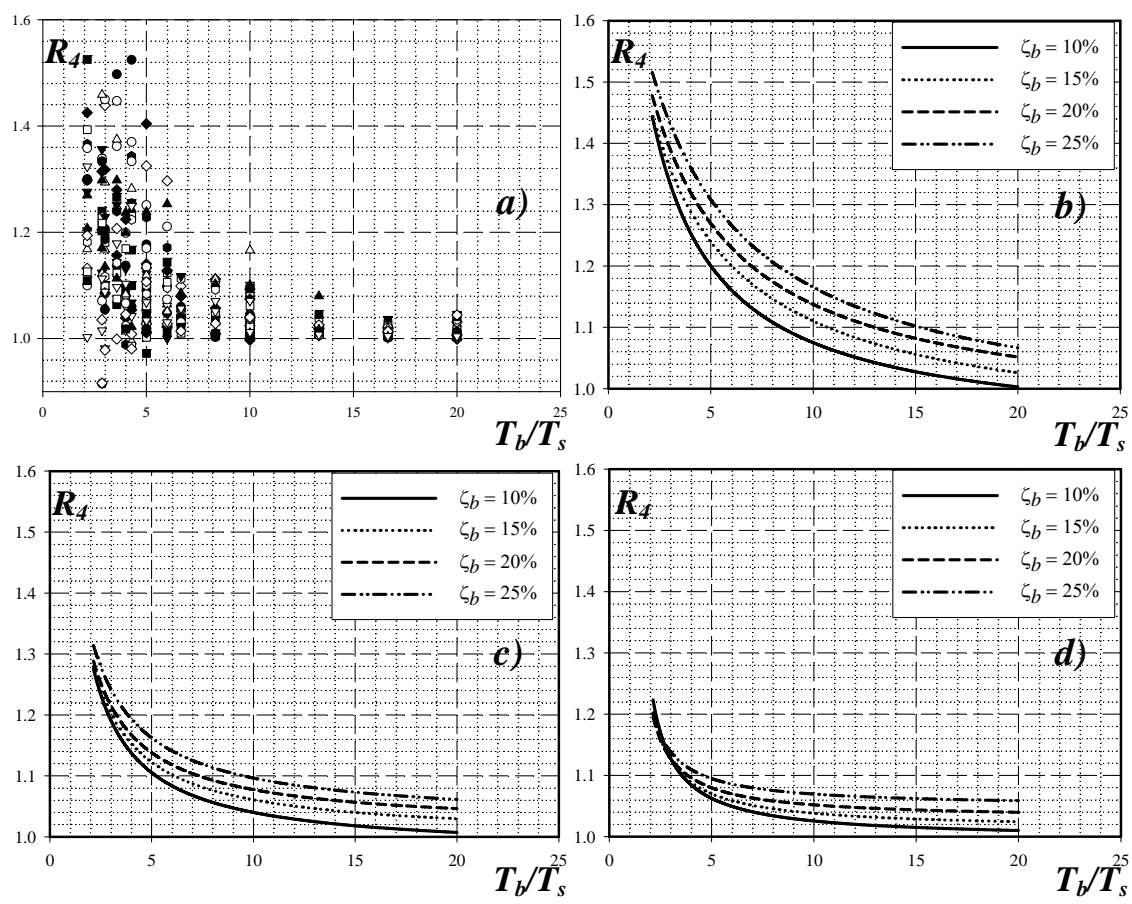

Figura 10. Cociente de aceleración para super-estructuras de cuatro pisos: a) Dispersión para $\zeta_{b}=0.10$ y $\gamma$ $=0.80$; b) Tendencias centrales para $\gamma=0.67$; c) Tendencias centrales para $\gamma=0.80$; d) Tendencias centrales para $\gamma=0.89$

\section{SISTEMA EQUIVALENTE PARA ESTRUCTURAS AISLADAS SÍSMICAMENTE}

Los espectros de respuesta son una fuente importante de información para la concepción de estructuras sismorresistentes (Bertero y Bertero 1992, Montiel y Terán 2011). Si el parámetro que define el desempeño sísmico de la estructura puede ser estimado razonablemente bien a partir de su periodo fundamental de vibración, un espectro puede utilizarse durante los estados preliminares del diseño para concebir un conjunto de propiedades estructurales globales que permitan a la estructura controlar adecuadamente su respuesta dinámica. Bajo este contexto, es de interés comparar la respuesta de un sistema aislado con sus correspondientes ordenadas espectrales para estudiar la posibilidad de establecer una metodología simple basada en aceleraciones para la concepción de estructuras aisladas.

Las figuras 11 y 12 superponen, respectivamente, la máxima aceleración absoluta en la base y azotea (normalizadas por la aceleración de la gravedad) de estructuras aisladas de cuatro niveles (super-estructuras con diferentes valores de $T_{s}$ ), y las ordenadas de espectros de pseudo-aceleración obtenidos para porcentajes de amortiguamiento crítico iguales a los sistemas de aislamiento (línea continua). Las abscisas de las demandas de aceleración absoluta corresponden al periodo de vibración del sistema de aislamiento $\left(T_{b}\right)$. En 
cuanto a las ordenadas espectrales y las demandas de aceleración absoluta ambas corresponden al valor medio de los valores obtenidos para los diferentes registros bajo consideración.

Una estimación razonable de la máxima demanda de aceleración absoluta en una estructura sismorresistente por lo general requiere de la consideración de por lo menos tres modos de vibrar. Sin embargo, el diseño adecuado de la rigidez lateral de la super-estructura (relativa a la del sistema de aislamiento) maximiza la contribución del modo fundamental de vibración, de tal manera que las demandas de aceleración absoluta en la base y azotea de la super-estructura pueden ser predichas con alta precisión a partir de un espectro de pseudo-aceleración. En estos términos, la situación ideal implica un sistema de aislamiento flexible con un bajo nivel de amortiguamiento y una super-estructura rígida. Para ilustrar esto, note en la figuras 11 y 12 que las demandas de aceleración que corresponden a los sistemas de aislamiento con $T_{b} / T_{s}$ igual o mayor que 10 (triángulos blancos) caen prácticamente sobre su correspondiente espectro de pseudo-aceleración. En el caso de super-estructuras flexibles, las demandas de aceleración absoluta pueden alejarse de forma considerable de sus respectivos espectros, de tal forma que no conviene plantear el uso de un sistema equivalente de un grado de libertad. Las figuras 11 y 12 muestran que un incremento en el nivel de amortiguamiento en el sistema de aislamiento hace más notable dicho alejamiento.
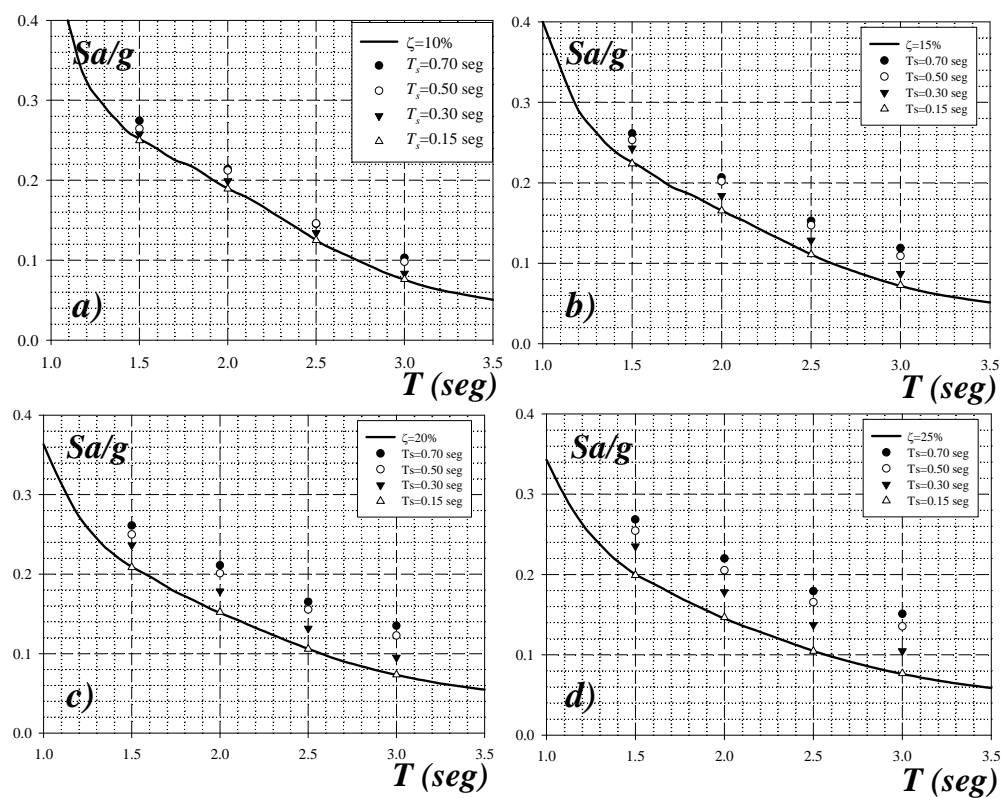

Figura 11. Comparación de demandas de aceleración absoluta en la base de super-estructuras de cuatro pisos con sus respectivas ordenadas espectrales: a) $\zeta_{b}=0.10$; b) $\zeta_{b}=0.15$; c) $\zeta_{b}=0.20$; d) $\zeta_{b}=0.25$

Una estimación razonable de las demandas de aceleración absoluta en los contenidos de sistemas aislados cuya respuesta este dominada por el modo fundamental de vibración, puede obtenerse a partir de una versión modificada del método discutido por Yasui et al. (1993):

$A_{e}\left(T_{e}, \zeta_{e}\right)=\frac{\sqrt{\left[\left(\frac{T_{b}}{T_{e}}\right)^{2} S_{a}\left(T_{b}, \zeta_{b}\right)\right]^{2}+S_{a}\left(T_{e}, \zeta_{e}\right)}}{\sqrt{1-\left(\frac{T_{b}}{T_{e}}\right)^{2}+4\left(\zeta_{b}+\zeta_{e}\right)^{2}\left(\frac{T_{b}}{T_{e}}\right)^{2}}}$ 
donde $A_{e}$ es la demanda de aceleración absoluta para contenidos elásticos con bajo amortiguamiento; $S_{a}$ es la ordenada espectral de pseudo-aceleración; $T_{e}$ y $T_{b}$ son los periodos del contenido y del sistema de aislamiento considerado como un sistema de un grado de libertad, respectivamente; y $\zeta_{e}$ y $\zeta_{b}$ son los porcentajes de amortiguamiento crítico correspondientes al contenido y sistema de aislamiento, respectivamente.
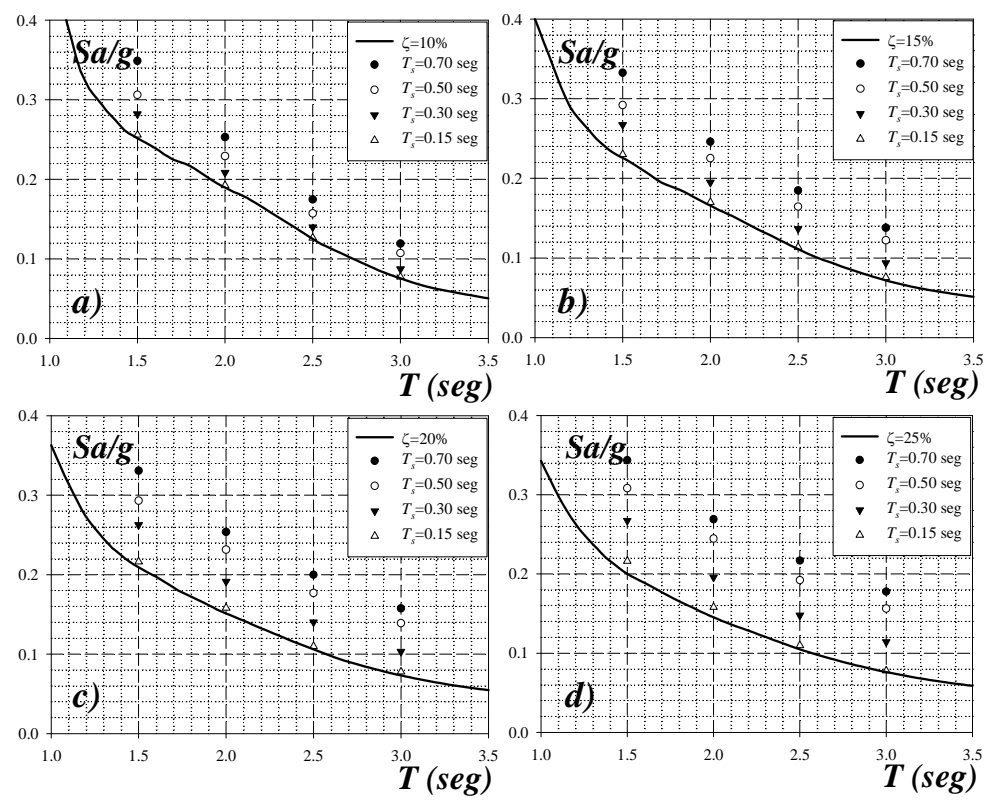

Figura 12. Comparación de demandas de aceleración absoluta en la azotea de super-estructuras de cuatro pisos con sus respectivas ordenadas espectrales: a) $\zeta_{b}=0.10$; b) $\zeta_{b}=0.15$; c) $\zeta_{b}=0.20$; d) $\zeta_{b}=0.25$

La figura 13 compara espectros de piso de aceleración con sus respectivas estimaciones obtenidas a partir de la ecuación 19. Conforme a lo esperado, se obtiene una buena aproximación para las demandas de aceleración para sistemas con relaciones de $T_{b} / T_{s}$ mayores que diez. En el caso de super-estructuras flexibles, $T_{b}$ no representa una buena forma de estimar el periodo fundamental de vibración de la estructura aislada (conforme lo ilustra la diferente localización de los picos en la figura 13d), y la ecuación 19 subestima, como se muestra en las figuras 13c y 13d, las demandas de aceleración para los contenidos que caen en el rango corto de periodos. Los resultados resumidos en las figuras 13a y 13b confirman el hecho de que un sistema estructural aislado se comporta como un sistema de un grado de libertad siempre y cuando se asigne una rigidez lateral lo suficientemente elevada a su super-estructura.

\section{OBSERVACIONES FINALES Y CONCLUSIONES}

Varios estudios analíticos y experimentales han demostrado la capacidad de los sistemas de aislamiento para reducir la respuesta dinámica de las estructuras sismorresistentes. A pesar de que el control del daño en los contenidos es una de las cualidades más atractivas de los sistemas estructurales aislados; en la actualidad no existen formatos de diseño por desempeño que permitan establecer las propiedades estructurales de este tipo de sistemas de tal forma que puedan controlar sus demandas de aceleración dentro de umbrales de daño bien definidos. 

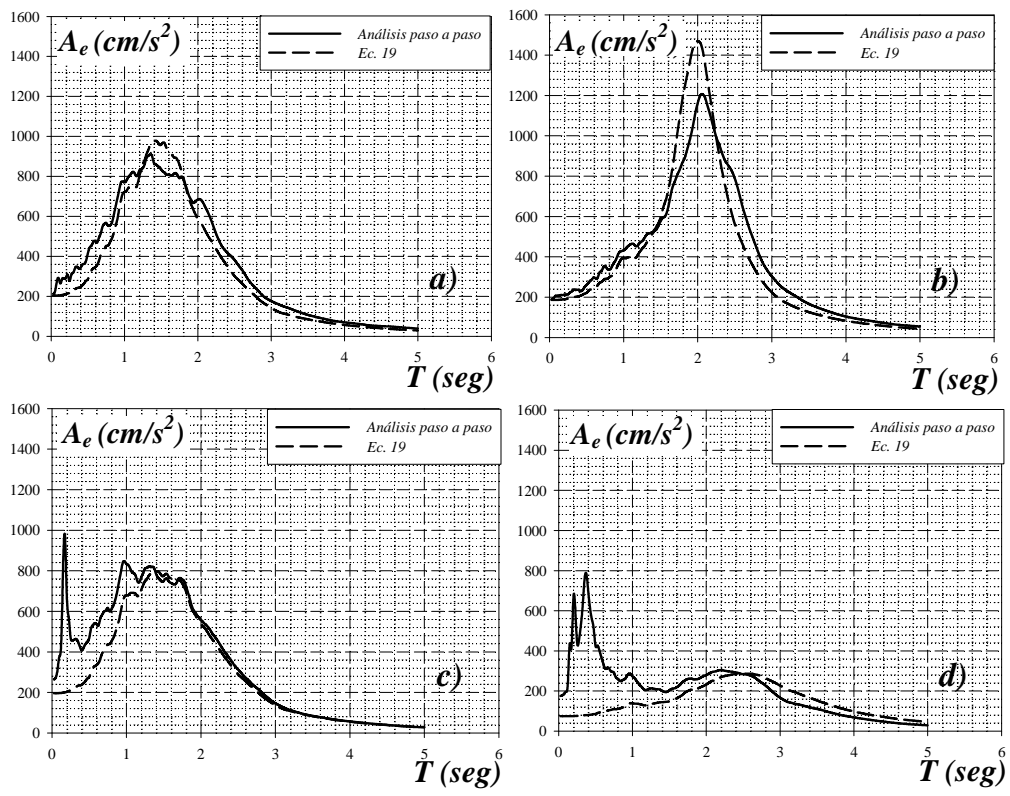

Figura 13. Comparación de espectro de aceleración absoluta de azotea con estimación obtenida con la ecuación 19: a) $T_{b}=1.5 \mathrm{seg}, T_{s}=0.15 \mathrm{seg}\left(T_{b} / T_{s}=10.0\right), \zeta_{b}=0.20$; b) $T_{b}=2.0 \mathrm{seg}, T_{s}=0.15 \mathrm{seg}$ ( $\left.T_{b} / T_{s}=13.33\right), \zeta_{b}=0.10$; c) $\left.T_{b}=1.5 \mathrm{seg}, T_{s}=0.30 \mathrm{seg}\left(T_{b} / T_{s}=5.0\right), \zeta_{b}=0.25 ; \mathrm{d}\right) T_{b}=3.0 \mathrm{seg}, T_{s}=0.70 \mathrm{seg}$

$$
\left(T_{b} / T_{s}=4.29\right), \zeta_{b}=0.25
$$

Aunque el aumento en el nivel de amortiguamiento en el sistema de aislamiento $\left(\zeta_{b}\right)$ tiende a incrementar la contribución de los modos superiores, el uso de sistemas estructurales con relaciones de $T_{b} / T_{s}$ entre 8 y 10 da como resultado un control adecuado de la contribución de dichos modos. Es importante notar que un cociente de $T_{b} / T_{s}$ de 8 es mucho mayor que el valor de tres sugerido en la literatura para promover un desempeño estructural adecuado de los sistemas estructurales aislados (Naeim y Kelly, 1999). El uso de porcentajes de amortiguamiento crítico en el sistema de aislamiento iguales o menores que $10 \%$ reducen los requisitos de rigidez lateral de la super-estructura, de tal manera que valores de $T_{b} / T_{s}$ mayores que seis pueden ser suficientes para controlar las demandas de aceleración. Aunque la masa de una estructura no puede ser considerada como un parámetro de diseño, los resultados mostrados en este artículo sugieren que una reducción de la masa en el nivel de aislamiento con respecto a la de la super-estructura conduce a una reducción en la contribución de los modos superiores. Por último, se concluye que la combinación de sistemas de aislamiento flexibles con bajos niveles de amortiguamiento con super-estructuras rígidas resulta en una reducción sustancial de la participación de los modos superiores a la respuesta global de las estructuras aisladas. Dentro de este contexto, es posible formular un modelo equivalente de un grado de libertad para la concepción de sistemas estructurales aislados en términos de aceleración.

El estudio de las demandas de desplazamiento que requieren satisfacer los sistemas aislados en el Pacífico Mexicano permite entender que estas no se constituyen en un problema para el diseño de uno de estos sistemas (tal como lo ilustra la figura 5b, las demandas máximas son del orden de $25 \mathrm{~cm}$ para sistemas con muy bajos niveles de amortiguamiento). Vale la pena mencionar que bajo ciertas circunstancias, el desplazamiento lateral en los aisladores puede ser un aspecto que controle el diseño sísmico, de tal manera que el control de la demanda de desplazamiento en el sistema de aislamiento puede llegar a limitar una concepción estructural basada en el control de las demandas de aceleración. 
El diseño de super-estructuras de baja altura que exhiban los valores de $T_{b} / T_{s}$ necesarios para controlar la respuesta de sus modos superiores no es un problema práctico. Por ejemplo, al considerar un sistema de aislamiento cuyo periodo oscile entre 2.0 hasta 3.0 segundos, un cociente $T_{b} / T_{s}$ de 6 implicaría una superestructura con $T_{s}$ entre 0.33 y 0.5 segundos. En el caso de super-estructuras de pocos niveles, no debiera haber problemas para alcanzar la rigidez lateral requerida por estos valores de $T_{s}$. En el caso de super-estructuras relativamente altas y esbeltas, será necesario revisar cuidadosamente la necesidad de proporcionar planos sismorresistentes rígidos que permitan reducir el período de la super-estructura. No obstante y de acuerdo con la teoría lineal discutida por Naeim y Kelly (1999), un aumento de la rigidez de la super-estructura debe traducirse en una reducción considerable en términos de sus demandas de fuerza y desplazamiento. Otro punto que debe enfatizarse es el hecho de que la reducción de los efectos de los modos superiores resulta en un comportamiento predecible de la super-estructura en términos de sus demandas de aceleración. En consecuencia, no sólo las demandas de aceleración son menores, sino la gran incertidumbre y complejidad observada en las demandas de sistemas sobre base rígida son prácticamente eliminadas.

Por último, es importante mencionar que el desarrollo de nuevos sistemas de aislamiento sísmico ha permitido reducir eficazmente las demandas sísmicas de los sistemas estructurales (Ahmadi y Su 1992, Fenz y Constantinou 2007, Johnson et al. 1998, Yoshioka et al. 2002). Aunque estos sistemas de aislamiento reducen las demandas de aceleración mucho más allá de las capacidades de un sistema tradicional de aislamiento, es posible decir que los conceptos desarrollados aquí son aplicables también a ellos. Los estudios resumidos en este documento son parte de un esfuerzo por implementar sistemas de aislamiento de bajo costo para clínicas hospitalarias de bajo costo y pocos niveles, ubicadas en la costa del Pacífico Mexicano. Dentro de este contexto, el uso de sistemas de aislamiento simples a base de caucho se ha considerado una prioridad.

\section{RECONOCIMIENTOS}

Los autores agradecen el apoyo de la Universidad Autónoma Metropolitana y al Consejo Nacional de Ciencia y Tecnología (CONACyT) por su apoyo durante la realización del presente trabajo de investigación.

\section{REFERENCIAS}

Adam, C., R. Heuer y A. Pirrotta. (2003), "Experimental Dynamic Analysis of Elastic-Plastic Shear Frames with Secondary Structures", Experimental Mechanics, 43(2), 124-30.

Ahmadi, G. y L. Su. (1992), "Equipment Response Spectra for Base-Isolated Shear Beam Structures", Nuclear Engineering and Design, 132(3), 287-308.

Bertero, V. V. y R. Bertero (1992), "Tall Reinforced Concrete Buildings: Conceptual EarthquakeResistant Design Methodology", Report No. UCB/EERC-92/16, Earthquake Engineering Research Center, UC Berkeley.

Chaudhuri, S. R. y T. C. Hutchinson. (2004), "Distribution of Peak Horizontal Floor Acceleration for Estimating Nonstructural Element Vulnerability", Proceedings of the 13th World Conference on Earthquake Engineering, (CD). Vancouver Canada: Artículo No. 1721.

Clark, P. W., M. Higashino y J. M. Kelly. (2002), "Performance of Seismically Isolated Structures in the January 17, 1994. Northridge Earthquake", University of California at Berkeley Earthquake Engineering Research Center, http://nisee.berkeley.edu/library/clark/peterclark.pdf. 
Clayton, J. y R. Medina. (2010). "Probabilistic Seismic Demand Evaluation of Acceleration-Sensitive Nonstructural Components Mounted on Structural Wall and Frame Structures" Proceedings of the 9th U.S. National/10th Canadian Conference on Earthquake Engineering, Toronto, CA, July 25-29.

Comerio, M. y W. Holmes. (2004), "Seismic Risk Reduction of Laboratory Contents", Proceedings of the 13th World Conference on Earthquake Engineering (CD). Vancouver, Canada: Artículo No. 3389.

Dowell, R. K., J. W. Smith y T. C. Hutchinson. (2008), "Floor and Attached Component Seismic Amplification Factors from Nonlinear Time-History Analysis," Proceedings of the 13th World Conference on Earthquake Engineering, (CD). Beijing, China: S20-031.

Elnashai, A. S., B. Gencturk, O.-S. Kwon, I. L. Al-Qadi, Y. Hashash, J. R. Roesler, S. J. Kim, S.-H. Jeong, J. Dukes y A. Valdivia. (2010), "The Maule (Chile) Earthquake of February 27, 2010: Consequence Assessment and Case Studies", Mid-America Earthquake Centre, Report No. 10-04, 190 páginas.

Fan, F.-G. y G. Ahmadi. (1992), "Seismic Responses of Secondary Systems in Base-Isolated Structures", Engineering Structures, 14(1), 35-48.

Fenz, D. y M. Constantinou. (2007), "Spherical Sliding Isolation Bearings with Adaptative Behavior: Theory", Earthquake Engineering \& Structural Dynamics, 37(2), 163-83.

Hurty, W. C. (1965), "Dynamic Analysis of Structural Systems Using Component Modes", AIAA Journal, 3(4), 678-85.

Igusa, T. (1990), "Response Characteristics of Inelastic 2-Dof Primary-Secondary System." Journal of Engineering Mechanics, 116(5), 1160-74.

Johnson, E., J. Ramallo, B. Spencer y M. Sain. (1998), "Intelligent Base Isolation Systems", Proceedings of the 2th World Conference on Structural Control (CD). Kyoto, Japón: 367-76.

Kaneko, M., Y. Nakamura, H. Kambara y K. Tamura. (2008), "Seismic Safety Evaluation Method for Buildings Contents", Proceedings of the 14th World Conference on Earthquake Engineering (CD). Beijing, China: s20-002.

Kelly, J. M. (1982), "The Influence of Base Isolation on the Seismic Response of Light Secondary Equipment." Report No. UCB/EERC-81/17, University of California at Berkeley.

Kelly, J. M. y H.-C. Tsai. (1985), "Seismic Response of Light Internal Equipment in Base-Isolated Structures", Earthquake Engineering \& Structural Dynamics, 13(6), 711-32.

Konstantinidis, D. y N. Markis. (2006), "Experimental and Analytical Studies on the Seismic Response of Freestanding and Restrained Laboratory Equipment", Proceedings of the 8th U.S. National Conference of Earthquake Engineering (CD). San Francisco C.A., U.S.A.: Artículo No. 1619.

Lin, J. y S. Mahin (1985), "Seismic Response of Light Subsystems on Inelastic Structures." Journal of Structural Engineering, 111(2), 400-17.

Marsantyo, R., T. Shimazu y H. Araki. (2000), "Dynamic Response of Nonstructural Systems Mounted on Floors of Buildings", Proceedings of the 12th World Conference on Earthquake Engineering (CD). Auckland, New Zeland: Artículo No. 1872.

Medina, R. A., J. Clayton, R. Sankaranarayanan y M. Ferguson. (2009), "Seismic Acceleration Demands on Nonstructural Components Attached to Elastic and Inelastic Structures", B. Goodno, ASCE, 5959. 
Medina, R. A., R. Sankaranarayanan y K. M. Kingston. (2006), "Floor Response Spectra for Light Components Mounted on Regular Moment-Resisting Frame Structures", Engineering Structures, 28(14), 1927-40.

Moehle, J. (1992), "Displacement-Based Design of RC Structures Subjected to Earthquakes", Earthquake Spectra, 8, 403-28.

Montiel-Ortega, M. y A. Teran-Gilmore. (2011), "Comparative Reliability of Two Twenty-Four Story Braced Buildings: Traditional Versus Innovative", The structural Design of Tall and Special Buildings, DOI:10.1002/TAL.716.

Naeim, F. y J. M. Kelly. (1999), "Design of Seismic Isolated Structures: From Theory to Practice", John Wiley \& Sons, New York, 297 páginas.

Poland, C. D. y D. B. Hom. (1997), "Opportunities and Pitfalls of Performance Based Seismic Engineering", Proceedings of Seismic Design Methodologies for the Next Generation Codes. Slovenia: 69-78.

Priestley, M., G. M. Calvi y M. J. Kowalsky. (2007), "Displacement-Based Seismic Design of Structures", IUSS Press, 1453-60 pp.

Retamales, R., G. Mosqueda, A. Filiatrault y A. M. Reinhorn. (2006), "Experimental Study on the Seismic Behavior of Nonstructural Components Subjected to Full-Scale Floor", Proceedings of the 8th National Conference on Earthquake Engineering (CD). San Francisco, CA.: Artículo No. 1359.

Rodriguez, M. E., J. I. Restrepo and J. J. Blandón (2007), "Seismic Design Forces for Rigid Floor Diaphragms in Precast Concrete Building Structures." Journal of Structural Engineering, 133(11), 1604-15.

Rojas, F., F. Naeim, M. Lew, L. D. Carpenter, N. F. Youssef, G. R. Saragoni y M. S. Adaros (2011), "Performance of Tall Buildings in Concepción During the 27 February 2010 Moment Magnitude 8.8 Offshore Maule, Chile Earthquake." The structural Design of Tall and Special Buildings, 20(1), 37-64.

Sankaranarayanan, R. (2007), "Seismic Response of Acceleration-Sensitive Nonstructural Components Mounted on Moment-Resistanting Frame Structures", Doctoral Thesis, Department of Civil and Environmental Engineering. College Park: University of Maryland, 203 pp, http://drum.lib.umd.edu/bitstream/1903/6700/1/umi-umd-4172.pdf.

Sankaranarayanan, R. y R. A. Medina. (2007), "Acceleration Response Modification Factors for Nonstructural Components Attached to Inelastic Moment-Resisting Frame Structures." Earthquake Engineering \& Structural Dynamics, 36(14), 2189-210.

Taghavi, S. y E. Miranda (2003), "Response Assessment of Nonstructural Building Elements", P. E. E. R. Center, PEER Report 2003/05, 84 páginas.

Takahashi, N. y H. Shiohara. (2004), "Life Cycle Economic Loss Due to Seismic Damage of Nonstructural Elements", Proceedings of the 13th World Conference on Earthquake Engineering (CD). Vancouver Canada: Artículo No. 203.

Todd, D., N. Carino, R. Chung, H. Lew, A. W. Taylor y W. D. Walton (1994), "1994 Northridge Earthquake: Performance of Structures, Lifelines and Fire Protection Systems." Gaithersburg, MD: U.S. Dept. of Commerce, National Institute of Standards and Technology, 173 pp.

Ventura, C. E., W. D. Liam Finn, J. F. Lord y N. Fujita (2003), "Dynamic Characteristics of a Base Isolated Building from Ambient Vibration Measurements and Low Level Earthquake Shaking." Soil Dynamics and Earthquake Engineering, 23(4), 313-22. 
Villaverde, R. (1997), "Seismic Design of Secondary Structures: State of the Art", Journal of Structural Engineering, 123(8), 1011-19.

Villaverde, R. (2006), "Simple Method to Estimate the Seismic Nonlinear Response of Nonstructural Components in Buildings", Engineering Structures, 28(8), 1209-21.

Yasui, Y., J. Yoshihara, T. Takeda y A. Miyamoto. (1993), "Direct Generation Method for Floor Response Spectra," Proceedings of the 12th International Conference SMiRT. Toronto, Canada: $\mathrm{K} 13 / 4$.

Yoshioka, H., J. Ramallo y B. Spencer. (2002), "Smart Base Isolation Strategies Employing Magnetorheological Dampers", Journal of Engineering Mechanics, 128(5), 540-51. 